\title{
AB-INITIO MODELING OF INTERMEDIATE BAND MATERIALS BASED ON METAL- DOPED CHALCOPYRITE COMPOUNDS
}

\author{
P. Wahnon , P. Palacios; K. Sanchez, I. Aguilera and J. C. Conesa
}

\begin{abstract}
Results of quantum calculations in M-doped chalcopyrite $\mathrm{Cu}_{4} \mathrm{MGa}_{3} \mathrm{~S}_{8}$ (with $\mathrm{M}=\mathrm{Ti}, \mathrm{V}, \mathrm{Cr}$ or $\mathrm{Mn}$ ) are evaluated. The purpose of this work is the quest of a compound which possesses an isolated narrow partiallyfilled electronic band sited into the host semiconductor bandgap. The aforementioned material could be useful for designing novel solar cells with very high efficiency. Density Functional Theory calculations in the spinpolarized GGA approach have been carried out in all cases for obtain band dispersion diagrams and densities of electronic states. For the systems having $\mathrm{Cr}$ and $\mathrm{Ti}$ as dopants, where the results reveal promising features, an advanced $\mathrm{DFT}+\mathrm{U}$ formalism has been used to ascertain their properties with higher certainty. Finally, after having reasoned that $\mathrm{Cu}_{4} \mathrm{TiGa}_{3} \mathrm{~S}_{8}$ has the desired features, a prediction of its energetic feasibility has been formulated.
\end{abstract}

\section{INTRODUCTION}

The intermediate band is a recent concept proposed for enhancing the efficiency of photovoltaic solar cells. It consists of a narrow, partially filled electronic band placed between the valence and conduction bands, dividing the former bandgap in two sub-bandgaps. A material with these features may absorb low energy photons in order to promote electrons from the valence to the intermediate band as well as from the latter to the conduction band [1]. In addition to this, conventional direct photoexcitations from valence to conduction band are also permitted.

With this, more efficient use of the light spectrum may produce an increase in photocurrent without decreasing the photovoltage. Consequently, a solar cell built with the intermediate band premises may reach an ideal efficiency above $63 \%$, substantially higher than the limiting efficiency of single junction solar cells (about $40 \%$ ).

In earlier work [2] it was stressed that a way of producing an intermediate band material is by means of doping a semiconductor with a transition metal. For instance, results of quantum calculations in Ti-doped $\mathrm{GaAs}$ and $\mathrm{GaP}$ compounds have shown the possible existence of the desired intermediate band [3]. In the present work $\mathrm{CuGaS}_{2}$ is chosen as host semiconductor candidate. This chalcopyrite compound has an analogous structure to CulnSe $e_{2}$, whose thin-films have been widely used in photovoltaic device applications. Chalcopyrite-type systems appear to have a big structural tolerance to a large range of anion-to-cation off-stoichiometry and can be doped $n$ and $p$ type by introduction of native defects or extrinsic impurities [4]. The present work is focused on the selective substitution of $\mathrm{Ga}$ atoms by $3 \mathrm{~d}$ metals, and assesses with quantum calculations the possible existence of an intermediate band in the thus formed $\mathrm{Cu}_{4} \mathrm{MGa}_{3} \mathrm{~S}_{8}$ compounds $(\mathrm{M}=\mathrm{Ti}, \mathrm{V}, \mathrm{Cr}$ or $\mathrm{Mn})$.

\section{MODELS AND METHODS}

The unit cell taken for all related calculations in the present work has been constructed from the vectors $(\mathbf{a}, \mathbf{b}, \mathbf{c})$ of a tetragonal $\mathrm{Cu}_{8} \mathrm{Ga}_{8} \mathrm{~S}_{16}$ cell (see Fig. 1) according to $\mathbf{a}^{\prime}=(\mathbf{a}+\mathbf{b}+\mathbf{c}) / 2, \mathbf{b}^{\prime}=(\mathbf{a}+\mathbf{b}-\mathbf{c}) / 2$ and $\mathbf{c}^{\prime}=\mathbf{b}-\mathbf{a}$. In the resulting 16 atom cell, one $\mathrm{Ga}$ atom has been replaced by one transition metal $\mathrm{M}$. The obtained $\mathrm{Cu}_{4} \mathrm{MGa}_{3} \mathrm{~S}_{8}$ cell is the primitive one of a centered monoclinic lattice with $\mathrm{C} 2$ symmetry. In this system the shortest $M-M$ distance is approximately $6.6 \AA$.

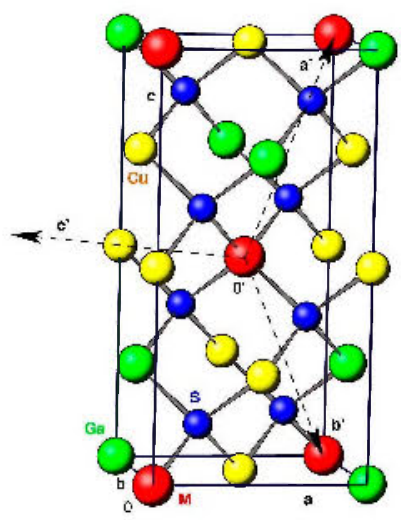

Fig. 1. Structure of the $\mathrm{Cu}_{4} \mathrm{MGa}_{3} \mathrm{~S}_{8}$ compound

In order to analyze the electronic structure of the compounds, quantum mechanics calculations based on DFT were carried out with the use of the plane-wave VASP code [5]. Ultrasoft pseudopotentials have been used and the GGA approach (in the PW91 formalism) has been taken for calculating the exchange-correlation 
contribution to the total energy. The Brillouin zone has been sampled using $6 \times 6 \times 6$ Monkhorst-Pack grids with 112 irreducible k-points including the gamma point.

Ionic relaxations without constraints were carried out with convergence tolerances of $0.01 \mathrm{eV} / \AA$ for atomic forces. Projections of DOS on spherical harmonics (to get orbital components) and local magnetic moments were obtained integrating inside atom-centered spheres having the standard atomic radii of the corresponding species.

Looking for the possibility of finding an isolated intermediate band inside of the semiconductor bandgap, a deeper study has been made for the most interesting cases, treating the electronic correlation in a more accurate way through inclusion of an effective on-site Coulomb parameter $U(G G A+U)$ applied over the $3 d$ electrons of the corresponding transition metal (the Hubbard approach). In addition, a prediction of the energetic feasibility of the candidate compounds is made through a comparison of their total energy with the addition of the energies of known compounds into which they may be decomposed.

\section{RESULTS}

Previously to the study of the M-doped systems, preliminary calculations for the host semiconductor $\mathrm{CuGaS}_{2}$ chalcopyrite were carried out. Comparing its density of states with the results obtained for a doped chalcopyrite is then a straightforward way of assessing how the substitution of one $\mathrm{Ga}$ atom by a transition metal has relevant consequences in the electronic structure (see, for example, the Ti-doped case in Fig. 2).

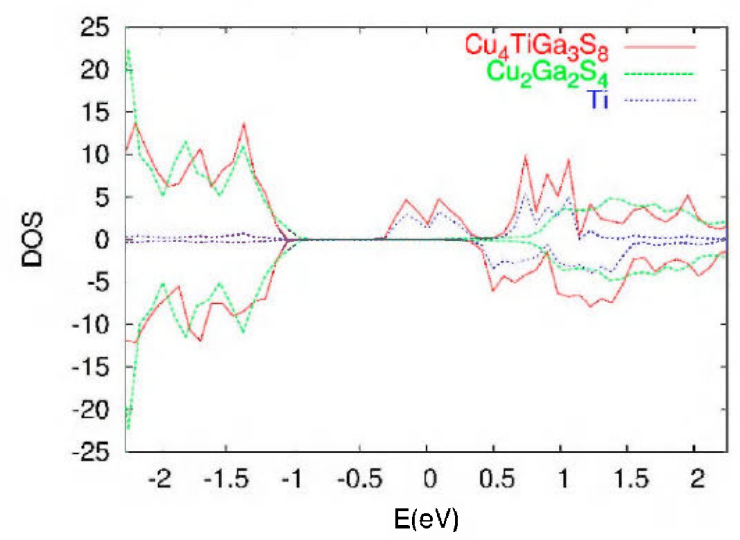

Fig. 2. Total and Ti-projected DOS for $\mathrm{Cu}_{4} \mathrm{TiGa}_{3} \mathrm{~S}_{8}$, compared with DOS for pure $\mathrm{CuGaS}_{2}$. Fermi level is set to zero, and both systems are aligned at the valence band.

It can be noticed (see Fig. 2 and Fig. 3 ) that the GGA result underestimates the bandgap of $\mathrm{CuGaS}_{2}$ (which is known to be $2.5 \mathrm{eV}$ ) by approximately $1.5 \mathrm{eV}$. An exact calculation of the electronic exchange energy, instead of an approximation for it as done in conventional DFT, should give a more accurate bandgap for $\mathrm{CuGaS}_{2}$. Hence, a calculation has been performed taking the EXX formalism implemented by our group in a numerical orbital basis set code called SIESTA [6]. The results (see Fig. 3) have shown an increase of the bandgap up to $1.5 \mathrm{eV}$. Unfortunately, EXX is a very time-consuming method and it was not yet possible to apply it for these doped compounds. Further work in this direction is planned.

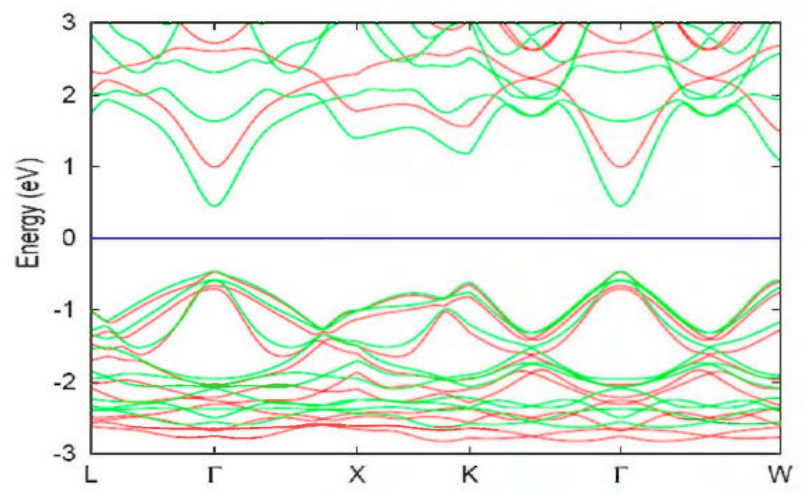

Fig. 3. Band dispersion curves for $\mathrm{CuGaS}_{2}$ compound. Red lines are obtained with EXX, green lines with GGA.

The transition metal substituting $\mathrm{Ga}$ in $\mathrm{CuGaS}_{2}$ behaves as a $\mathrm{M}^{3+}$ ion, and the near-regular tetrahedral coordination in which it is introduced splits its $3 \mathrm{~d}$ electronic states (for each spin polarization) in two main manifolds. The lower energy manifold has $e_{g}$ symmetry and is doubly degenerated. The higher one has $t_{2 g}$ symmetry and is triply degenerated. It is expected that the desired intermediate band may be produced by one of these manifolds. Results of GGA calculations support these arguments as is explained below.

For $\mathrm{Ti}$ substitution the formed $\mathrm{Ti}^{3+}$ ion has only one electron available for filling the bands derived from the split manifolds. Therefore the electron fills partially the corresponding $\mathrm{e}_{\mathrm{g}}{ }^{+}$band (the + sign stands for majority spin), which is crossed by the Fermi level (see Fig. 4).

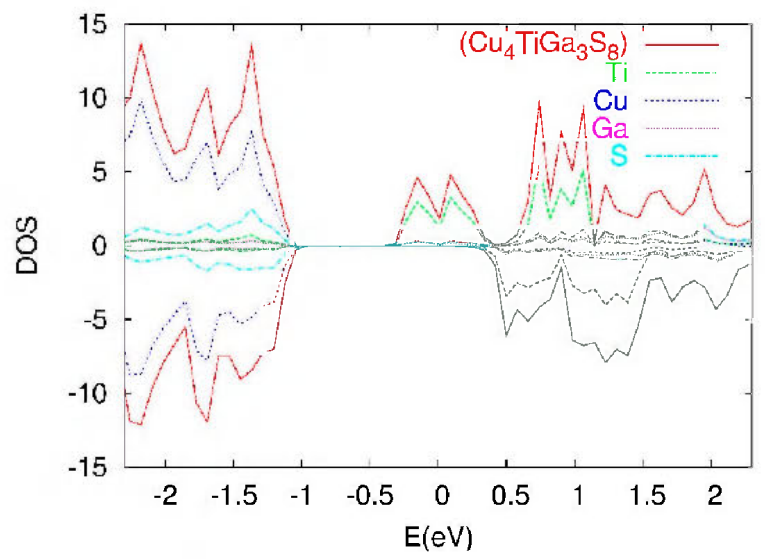

Fig. 4. Total and projected DOS curves for $\mathrm{Cu}_{4} \mathrm{TiGa}_{3} \mathrm{~S}_{8}$.

This band is clearly separated (more than $0.5 \mathrm{eV}$ ) from the valence band but overlaps slightly the conduction one. 
As explained below this overlap is not definitive, and although from this GGA result $\mathrm{Cu}_{4} \mathrm{TiGa}_{3} \mathrm{~S}_{8}$ seems to be a metal, a deeper study is considered necessary.

The $\mathrm{Cu}_{4} \mathrm{VGa}_{3} \mathrm{~S}_{8}$ compound is expected to be unsuitable as candidate for having an intermediate band. The two remaining electrons of the $V^{3+}$ cation fill completely the $\mathrm{e}_{\mathrm{g}}{ }^{+}$manifold while the $\mathrm{t}_{2 \mathrm{~g}}{ }^{+}$levels are empty As a result this compound shows an insulator behavior.

In the case of $\mathrm{Cr}$-doped $\mathrm{CuGaS}_{2}$ a partially filled band (with occupation 1/3), which now it consists of $3 d-t_{2 g}{ }^{+}$ states of chromium, appears isolated inside the bandgap. This is consistent with the projected density of states curve (see Fig. 5) where this band has a predominant population located around the $\mathrm{Cr}$ atom.

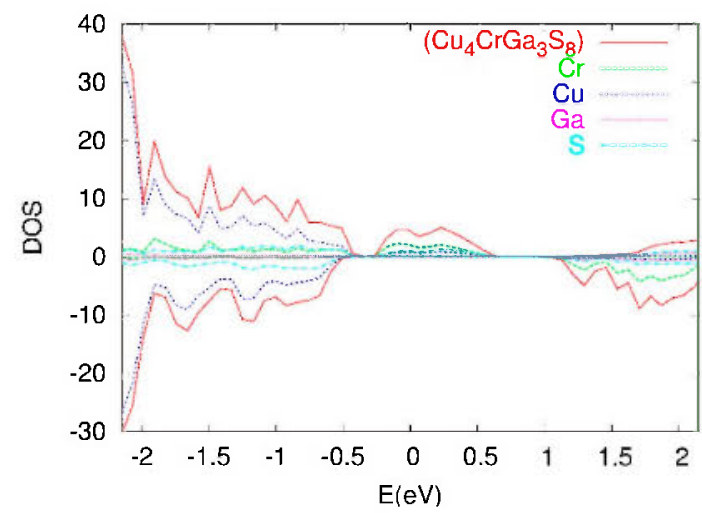

Fig. 5. Total and projected DOS curves for $\mathrm{Cu}_{4} \mathrm{CrGa}_{3} \mathrm{~S}_{8}$.

Finally, results from $\mathrm{Cu}_{4} \mathrm{MnGa}_{3} \mathrm{~S}_{8}$ compound show it is a metal. Because of the high electronegativity of $\mathrm{Mn}$ its majority $3 \mathrm{~d}$ levels are excessively low in energy and overlap strongly with the former valence band of $\mathrm{CuGaS}_{2}$.

In summary, after the GGA study, $\mathrm{Cr}$ and $\mathrm{Ti}$ offer probabilities for forming a suitable intermediate band material when doping $\mathrm{CuGaS}_{2}$, whereas $\mathrm{V}$ and $\mathrm{Mn}$ are rejected as they do not accomplish this goal.

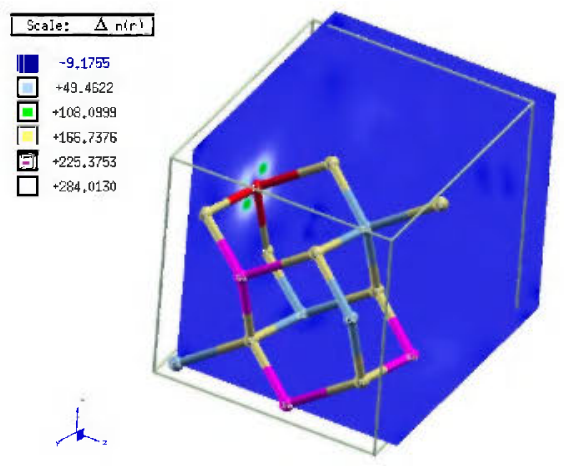

Fig. 6. Spin density distribution $\left(n^{+}-n^{-}\right)$for $\mathrm{Cu}_{4} \mathrm{TiGa}_{3} \mathrm{~S}_{8}$.

Besides all the interesting results explained above, it is worthwhile mentioning that $3 \mathrm{~d}$-element split manifolds, as expected, present a full spin polarization. This can be seen from the projected DOS curves (see Fig. 4, Fig. 5) where the components appearing at energies within the forbidden gap of the $\mathrm{CuGaS}_{2}$ semiconductor are fully spinpolarized. Furthermore, spin-density charts (see Fig. 6 and Fig. 7) reveal that the spin-polarization is centered in the $\mathrm{M}$ atoms and has a typical d-orbital shape. Although this fact is not relevant for photovoltaic purposes, it could have applications in other fields as, for instance, spintronics.

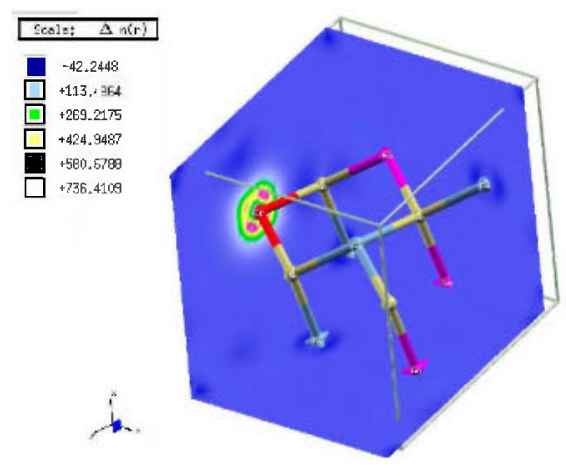

Fig. 7 Spin density distribution $\left(n^{-}-n^{-}\right)$for $\mathrm{Cu}_{4} \mathrm{CrGa}_{3} \mathrm{~S}_{8}$.

LSDA and GGA approaches, which predict the exchange and correlation energy with a mean-field formalism, have difficulties in treating properly systems with highly localized electrons because of their strong correlation. This is the case of $3 d$ electrons in M-doped chalcopyrites. To overcome this problem, correction terms have been applied to our GGA calculations for $\mathrm{Cu}_{4} \mathrm{CrGa}_{3} \mathrm{~S}_{8}$ and $\mathrm{Cu}_{4} \mathrm{TiGa}_{3} \mathrm{~S}_{8}$, whose results may change notably when an accurate description of the correlation is taken into account. The values used for the Hubbard term $\mathrm{U}$ have been $5 \mathrm{eV}$ and $2 \mathrm{eV}$ for $\mathrm{Cr}$ and $\mathrm{Ti}$, respectively.

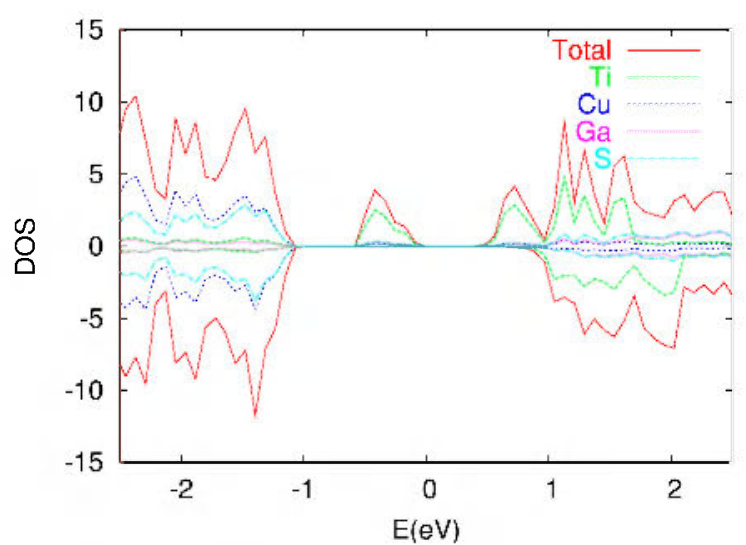

Fig. 8. Total and projected DOS curves for $\mathrm{Cu}_{4} \mathrm{TiGa}_{3} \mathrm{~S}_{8}$ computed with the GGA+U approach.

When the $U$ correction is applied to the Ti-doped chalcopyrite calculations, the $\mathrm{e}_{\mathrm{g}}^{+}$manifold is separated completely from the conduction band (see Fig. 8) and the compound turns out to have an isolated band placed inside the bandgap. From these more reliable results it 
can be concluded that $\mathrm{Cu}_{4} \mathrm{TiGa}_{3} \mathrm{~S}_{8}$ have the adequate characteristics for become an intermediate band material.

However, the GGA+U study of $\mathrm{Cu}_{4} \mathrm{CrGa}_{3} \mathrm{~S}_{8}$ (see Fig 9) corrects the density of states given by the initial GGA calculation (see Fig. 5) and predicts that this material has not the previously anticipated intermediate band. The additional splitting made by the $U$ parameter pushes down the $\mathrm{t}_{2 \mathrm{~g}}{ }^{+}$manifold that now overlaps the valence band, resulting in an electronic structure that is inappropriate for the purposes of this work.

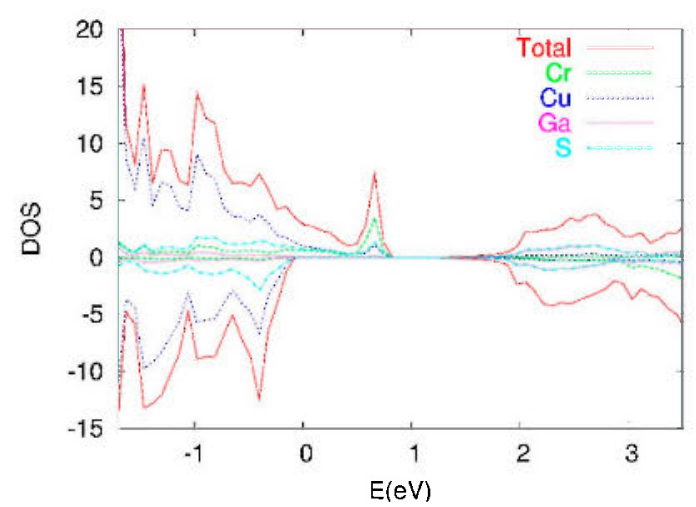

Fig. 9. Total and projected DOS curves for $\mathrm{Cu}_{4} \mathrm{CrGa}_{3} \mathrm{~S}_{8}$ computed with the GGA+U approach.

After having determined that $\mathrm{CuTi}_{x} \mathrm{Ga}_{1-x} \mathrm{~S}_{2}$ is a compound with suitable electronic characteristics, a prediction of its energetic feasibility has been carried out as stated below. Its instability would depend on the energetic balance of a decomposition into the most likely known compounds, that in this case would probably be:

$$
\mathrm{Cu}_{4} \mathrm{TiGa}_{3} \mathrm{~S}_{8} \rightarrow 3 \mathrm{CuGaS}_{2}+\mathrm{Cu}_{2 / 3} \mathrm{TiS}_{2}+1 / 3 \mathrm{Cu}
$$

The total energy balance computed for this process is $0.82 \mathrm{eV}$ per $\mathrm{Ti}$ atom. Although favorable to the decomposition, it has a moderate value when compared to similar calculations carried out for other compounds [7]

\section{CONCLUSIONS}

Quantum calculations in the framework of the Density Functional Theory have been carried out for $\mathrm{Cu}_{4} \mathrm{MGa}_{3} \mathrm{~S}_{8}$ systems (with $\mathrm{M}=\mathrm{Ti}, \mathrm{V}, \mathrm{Cr}$ or $\mathrm{Mn}$ ) in order to obtain their electronic properties.

Results of GGA calculations have shown certainly that $\mathrm{V}$ and $\mathrm{Mn}$ are not suitable doping species for our purposes. Initially, the $\mathrm{Cu}_{4} \mathrm{CrGa}_{3} \mathrm{~S}_{8}$ compound seems to present an electronic intermediate band with the desired features, whereas the Ti-doped chalcopyrite would seem not to contain it. Nevertheless, these cases are at the edge of what can be computed correctly with this level of theory, and therefore a study providing a better account of the strong correlations existing between the $3 d$ electrons of the transition metals is necessary.
More accurate calculations made with the $G G A+U$ approach predict finally that only $\mathrm{Cu}_{4} \mathrm{TiGa}_{3} \mathrm{~S}_{8}$ compound presents an isolated intermediate band.

An energetic prediction of the stability of the candidate compound based on the computed total energies indicates that it is not stable. However, its production may seem feasible once this result is compared with those obtained for other cases known to allow such dopings.

\section{ACKNOWLEDGEMENT}

We acknowledge funding from project FULLSPECTRUM (contract. SES-CT-2003-502620) of the $6^{\text {th }}$ European Framework Programme, from the Spanish National R\&D contract MAT2004-04631 and from the Community of Madrid Research Programme "NUMANCIAMA" (S-05050/ENE/0310).

\section{REFERENCES}

[1] A. Luque and A. Marti, "Increasing the Efficiency of Ideal Solar Cells by Photon Induced Transitions at Intermediate Levels", Phys. Rev. Letters 78, 1997, pp. 5014-5017.

[2] P. Wahnon and C. Tablero," $A b$ initio electronic structure calculations for metallic intermediate band formation in photovoltaic materials" Phys. Rev. B 65, 2002, 165115. P. Wahnon, P. Palacios, J. J. Fernandez and C. Tablero," Ab-initio spin polarized electronic structure calculations for $\mathrm{Ti}_{x} \mathrm{Ga}_{n} A s_{m}$ photovoltaic materials" J. Maters. Sci. 40, 2005, pp. 1383-1386.

[3] P. Palacios, J. J. Fernandez, K. Sanchez, J. C. Conesa and $P$. Wahnon, "First principles investigation of isolated band formation in half-metallic $\mathrm{Ti}_{x} \mathrm{Ga}_{1-\mathrm{x}} \mathrm{P}(\mathrm{x}=0.3125-0.25)$ ", Phys. Rev. B 73, 2006, 085206.

[4] P. Palacios, K. Sanchez, J. C. Conesa and P. Wahnón, "First principles calculation of isolated intermediate bands formation in a transition metal chalcopyrite semiconductor", Phys. Stat. Sol. (a) 203, 2006, pp. 13951401.

[5] G. Kresse and J. Hafner," Ab initio molecular dynamics for liquid metals" Phys, Rev, B 47, 1993, pp. 558-561. G. Kresse and J. Furthmuller, "Efficient iterative schemes for $a b$ initio total-energy calculations using a plane-wave basis set", Phys. Rev. B 54, 1996, pp. 11169-11186.

[6] J. J. Fernandez, C. Tablero and P. Wahnón, "Development and implementation of the exact exchange method for semiconductors using a localized basis set", Comp. Mat. Sci. 28, 2003, pp. 274-286.

[7] P. Palacios, P. Wahnon, S. Pizzinato and J. C. Conesa, "Energetics of Formation of $\mathrm{TiGa}_{3} \mathrm{As}_{4}$ And $\mathrm{TiGa}_{3} \mathrm{P}_{4}$ Intermediate Band Materials" J. Chem. Phys. 124, 2006, 014711. 